\title{
Proton-irradiation-induced intermixing of InGaAs quantum dots
}

\author{
P. Lever, ${ }^{\text {a) }}$ H. H. Tan, and C. Jagadish \\ Department of Electronic Materials Engineering, Research School of Physical Sciences and Engineering, \\ The Australian National University, Canberra, ACT 0200, Australia \\ P. Reece and M. Gal \\ School of Physics University of New South Wales, Sydney, NSW 2052, Australia
}

(Received 4 November 2002; accepted 20 January 2003)

\begin{abstract}
Proton irradiation was used to create interdiffusion in $\mathrm{In}_{0.5} \mathrm{Ga}_{0.5} \mathrm{As}$ quantum dots (QDs), grown by low-pressure metalorganic chemical vapor deposition. After $25-\mathrm{keV}$ proton irradiation, the QD samples were annealed at two temperatures $\left(700\right.$ or $\left.750{ }^{\circ} \mathrm{C}\right)$ for $30 \mathrm{~s}$. It was found that much lower annealing temperatures were needed to recover the photoluminescence signals than in the quantum-well case. Large blueshifts $(120 \mathrm{meV})$ and narrowing of the photoluminescence spectra were seen. Various doses $\left(5 \times 10^{13}-1 \times 10^{15} \mathrm{~cm}^{-2}\right)$ and implant temperatures $\left(20-200^{\circ} \mathrm{C}\right)$ were used to study the interdiffusion processes in these samples. In QD samples, much lower doses were required to achieve similar energy shifts than reported in quantum-well samples. () 2003 American Institute of Physics. [DOI: 10.1063/1.1561153]
\end{abstract}

There has been a lot of interest in the study of quantum dots (QDs) due to the three-dimensional confinement of carriers leading to an atom-like density of states. QD lasers ${ }^{1}$ and photodetectors $^{2}$ with improved characteristics have already been demonstrated. Intermixing techniques allow postgrowth tuning of the emission wavelength. Using these techniques, lasers of different wavelengths can be fabricated on the same chip for wavelength division multiplexing applications. The control of the band gap in various regions of a chip is critical for the fabrication of photonic integrated circuits. There are several methods to achieve this. Impurityfree vacancy disordering uses dielectric caps to create vacancies in selected regions, while ion implantation induced intermixing creates vacancies through bombardment. In studies on QD intermixing annealing, with and without dielectric capping, has been shown to cause a large blueshift and significant narrowing in the QD photoluminescence (PL) spectra. ${ }^{3,4}$ In this process, interdiffusion occurs between the GaAs barrier layers and the InGaAs or InAs QDs, leading to the confining potential of the QDs becoming shallower. However, there have been studies indicating that if too much interdiffusion takes place, the QDs merge into a quantum well. ${ }^{5}$

Ion-implantation-induced intermixing is a widely used technique for modifying the potential profile of a quantum well and quantum-well lasers. ${ }^{6-9}$ Ion implantation has several advantages over other techniques used for intermixing. Ion implantation is compatible to planar processing as one can accurately control the concentration and depth distribution of the defects by varying the ion dose and energy. This will allow creation of controlled interdiffusion in QDs in selected regions.

Although the interdiffusion in QDs by thermal annealing has been widely studied, there have only been a few studies on ion-implantation-induced interdiffusion. These studies used medium-mass ions $(\mathrm{Mn} \text { and } \mathrm{Cr})^{10,11}$ and were done on

${ }^{a)}$ Electronic mail: penny_lever@ieee.org
InAs dots grown by molecular beam epitaxy. The QD composition, growth methods, and implant conditions are expected to play a major role in the interdiffusion of the QDs. In this work, proton irradiation was used on a single layer of $\mathrm{In}_{0.5} \mathrm{Ga}_{0.5} \mathrm{As}$ QDs, grown by metalorganic chemical vapor deposition (MOCVD). Based on previous studies of proton irradiation into quantum wells, ${ }^{7}$ these light ions create dilute cascades that lead to generation of a large concentration of point defects and hence a large degree of interdiffusion. Proton irradiation was carried out with various doses and temperatures. Low-temperature PL spectra were measured and used to characterize the interdiffusion created in the samples.

$\mathrm{In}_{0.5} \mathrm{Ga}_{0.5}$ As QDs were grown by low-pressure MOCVD. A buffer layer of $\mathrm{GaAs}$ was grown at $650{ }^{\circ} \mathrm{C}$, then the growth temperature was reduced to $550{ }^{\circ} \mathrm{C}$ for the dot growth. A total of 6 monolayers (ML) of material was used to form the dots. After the dot growth, the temperature was ramped up to $650{ }^{\circ} \mathrm{C}$ during the GaAs capping layer growth. A 200-nm GaAs cap was grown on top of the dots. Atomic force microscopy was used on uncapped samples grown under the same conditions to measure the size and density of the dots. The dots are 3-5 $\mathrm{nm}$ high and $20-30 \mathrm{~nm}$ wide, with a density of $3.6 \times 10^{10} \mathrm{dots} / \mathrm{cm}^{2}$.

After growth, samples were irradiated with $\mathrm{H}$ ions at 25 $\mathrm{keV}$ to doses of $5 \times 10^{13}$ to $1 \times 10^{15} \mathrm{~cm}^{-2}$ at temperatures ranging from RT to $200^{\circ} \mathrm{C}$. The energy of the ions was chosen to position the damage peak over the QD region. After implantation, the samples were annealed to remove the damage caused by implantation and initiate the interdiffusion process. This was carried out in a rapid thermal annealer under $\mathrm{Ar}$ ambient. The samples were proximity capped and annealed at 700 or $750{ }^{\circ} \mathrm{C}$ for $30 \mathrm{~s}$. For PL measurement, the samples were excited with the 514.5-nm line of an Ar-ion laser and the spectra were collected using a liquid-nitrogencooled Ge detector.

Thermal interdiffusion in QD samples is a large effect. An energy shift of $143 \mathrm{meV}$ is seen in the luminescence peak of the sample used in this study when it is annealed at $850{ }^{\circ} \mathrm{C}$ 


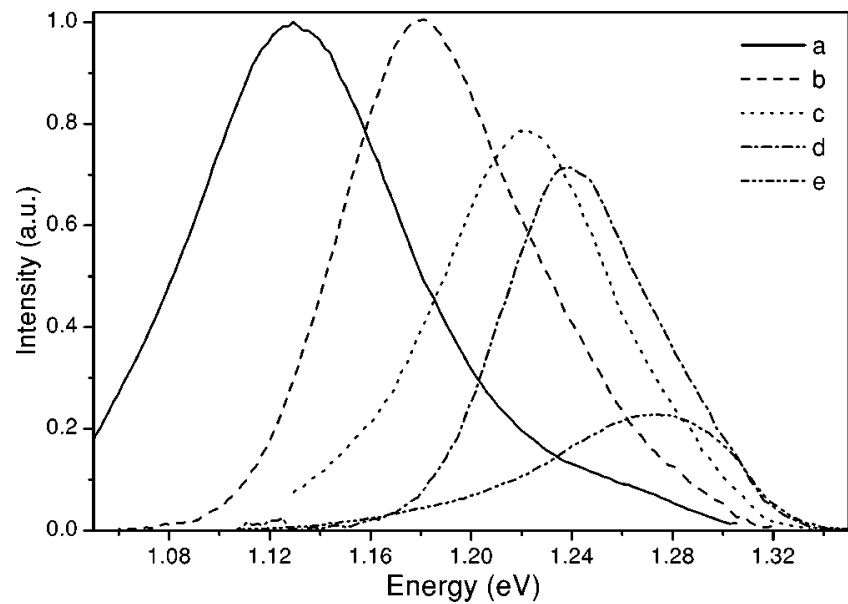

FIG. 1. PL spectra measured at $10 \mathrm{~K}$ from proton-implanted and annealed (at $750{ }^{\circ} \mathrm{C}$ for $30 \mathrm{~s}$ ) QD samples: (a) as-grown, (b) annealed with no implantation, (c) $5 \times 10^{13} \mathrm{H} / \mathrm{cm}^{2}, \quad$ (d) $1 \times 10^{14} \mathrm{H} / \mathrm{cm}^{2}, \quad$ and (e) 1 $\times 10^{15} \mathrm{H} / \mathrm{cm}^{2}$.

for $30 \mathrm{~s}$. The linewidth was reduced to $59 \%$ of the original value. With so much interdiffusion occurring due to thermal energy, there are concerns that dissolution of the dots ${ }^{5}$ can take place. Strain relaxation could also be a factor. In this study, the annealing temperatures are kept as low as possible to minimize the effect of thermal interdiffusion.

Annealing at $700{ }^{\circ} \mathrm{C}$ was found to be sufficient to recover the PL intensity to more than half of the original value for the lowest dose. Figure 1 shows the PL spectra from the QD sample annealed at $750^{\circ} \mathrm{C}$ for $30 \mathrm{~s}$. The energy shift from the as-grown to the annealed (unimplanted) sample is $45 \mathrm{meV}$. The additional energy shift from implantation is from 40 to $75 \mathrm{meV}$, depending on the implantation dose. The PL intensity from the lowest dose implant returns to $76 \%$ of its original value in the unimplanted sample. The PL intensity of the highest dose sample recovered to $22 \%$ of its original value. Even annealing at $900{ }^{\circ} \mathrm{C}$, InGaAs/GaAs quantum wells implanted with the same dose have PL intensity of less than one tenth of the original value. ${ }^{12}$ The energy shifts seen in this study lie between the energy shifts seen in two other studies $^{10,11}$ when compared against the amount of damage created in the sample. There are several differences that can be expected in the behavior of the samples previously reported in literature and the $\operatorname{In}_{0.5} \mathrm{Ga}_{0.5}$ As QDs grown in this study. The InAs QDs used in the previous studies should show a larger effect from interdiffusion as they have a larger In concentration gradient to cause interdiffusion. However, all these QDs are highly strained, and during the interdiffusion process this strain could partially relax. As the InAs dots have more strain than the $\operatorname{In}_{0.5} \mathrm{Ga}_{0.5}$ As dots, they are more likely to show the strain relaxation effects.

Two different annealing temperatures were used after the implant, resulting in different amounts of energy shift. In Fig. 2, the implantation-induced energy shift is plotted for the different annealing temperatures. The implantationinduced energy shift is taken to be the difference between the sample that has been annealed but not implanted and the one that has been implanted and annealed. As noted in this figure, there is less implantation-induced energy shift at the higher annealing temperatures. However, the overall energy shift was larger after annealing at the higher temperature. The Downloaded 14 Apr 2010 to 150.203.243.38. Redistribution subje

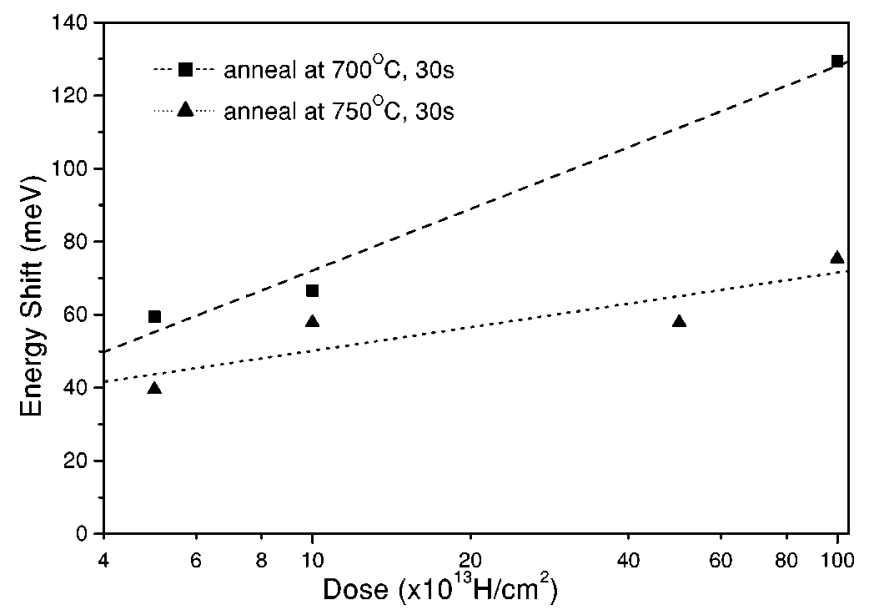

FIG. 2. The implantation-induced PL energy shift after annealing at different annealing temperatures.

larger thermal interdiffusion at the higher temperature leads to smaller differential energy shifts.

The linewidth reduction in the luminescence spectra is proportional to the amount of blueshift. There could be several factors involved in the reduction of the PL linewidth. The shape of the potential profile is changed as the interface becomes graded. As-grown dots are known to have an inhomogeneous In distribution, and at the areas of high In, more interdiffusion will take place. This will lead to a smooth potential profile and will also homogenize the dots. Another factor is that the energy shift from as-grown to interdiffused quantum wells has been shown to be dependent on the thickness of the quantum well. ${ }^{13}$ This is due to the position of the energy levels within the as-grown well with respect to the barrier level. It follows that this would also occur in QDs and could contribute to the narrowing of the PL spectra. The narrowing of the linewidth from the implanted samples is slightly less than the linewidth reduction for the samples annealed without implantation. This is expected, as the broadening due to residual defects introduced by implantation offsets the narrowing gained from the added interdiffusion.

The effect of different implantation temperatures was also studied. The implantations were carried out using the two highest doses at 20,100 , and $200^{\circ} \mathrm{C}$. The amount of energy shift seen after annealing at $750{ }^{\circ} \mathrm{C}$ for $30 \mathrm{~s}$ is shown in Fig. 3. As the implantation temperature increases the amount of implantation induced interdiffusion decreases. The implantation induced blueshift is $75 \mathrm{meV}$ at room temperature, but only $19 \mathrm{meV}$ at $200{ }^{\circ} \mathrm{C}$ for the highest dose ( 1 $\times 10^{15} \mathrm{~cm}^{-2}$ ). The luminescence intensities in the samples implanted at the elevated temperatures are also much brighter. Both these effects can be attributed to dynamic annealing processes occurring during the implants at elevated temperatures. This has been seen previously in $\mathrm{InGaAs} / \mathrm{GaAs}$ quantum wells. ${ }^{12}$ The interdiffusion process is controlled by the concentration of point defects present before annealing. Since proton implantation creates dilute defect cascades, there could be significant annihilation of vacancies and interstitials. This process occurs even at room temperature, but is enhanced at the elevated implant temperatures. Hence, the concentration of residual point defects after elevated temo AIP license or copyright; see http://apl.aip.org/apl/copyright.jsp 


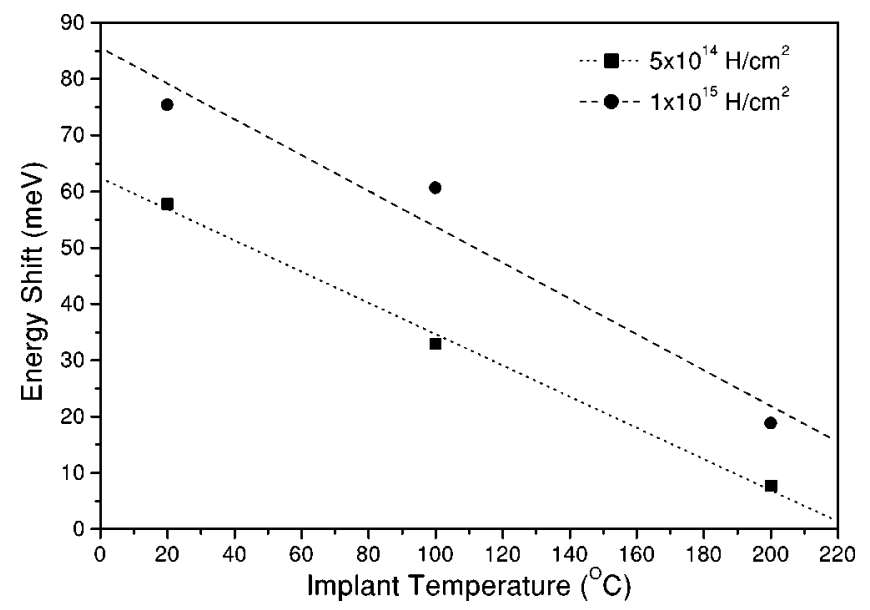

FIG. 3. The implantation-induced PL energy shift after implantation at various temperatures, for different proton doses. Samples were annealed at $750{ }^{\circ} \mathrm{C}$ for $30 \mathrm{~s}$.

perature implantation is less than after implantation at room temperature. As the degree of interdiffusion is related to the concentration of point defects, less interdiffusion is observed as the temperature is increased. Another process that could occur at elevated temperatures is the clustering of defects into large clusters or loops. These would lead to large nonradiative recombination centers; if they were present in the samples, the intensity of the luminescence would be weaker. However, in our case, we see brighter luminescence at the elevated temperatures than in the room temperature case. Therefore, the reduction in interdiffusion must be related to the dynamic annealing occurring at the elevated temperatures.

In summary, the interdiffusion processes in InGaAs QDs caused by proton irradiation and annealing were studied. PL energy shifts were found to increase with proton dose for the doses studied. It was found that the PL intensity recovered much better in $\mathrm{In}_{0.5} \mathrm{Ga}_{0.5}$ As QD samples than has been reported for InGaAs quantum wells. Annealing even at $750{ }^{\circ} \mathrm{C}$ for $30 \mathrm{~s}$ has been found to be sufficient to recover more than $20 \%$ of the original PL intensity, even for the highest dose used in this study. Implantation at elevated temperatures reduced the amount of PL energy shift, but enhanced recovery of the luminescence was observed.

${ }^{1}$ D. Bimberg, M. Grundmann, F. Heinrichsdorff, N. Ledentsov, V. Ustinov, A. Zhukov, A. R. Kovsh, M. Maximov, Y. Shernyakov, B. Volovik, A. Tsatsul'nikov, P. Kop'ev, and Z. I. Alferov, Thin Solid Films 367, 235 (2000).

${ }^{2}$ A. Stiff-Roberts, S. Krishna, P. Bhattacharya, and S. Kennerly, J. Vac. Sci. Technol. B 20, 1185 (2002).

${ }^{3}$ R. Leon, Y. Kim, C. Jagadish, M. Gal, J. Zou, and D. J. H. Cockayne, Appl. Phys. Lett. 69, 1888 (1996).

${ }^{4}$ D. Bhattacharyya, A. S. Helmy, A. C. Bryce, E. A. Avrutin, and J. H. Marsh, J. Appl. Phys. 88, 4619 (2000).

${ }^{5}$ A. Babinski, J. Jasinski, R. Bozek, A. Szepielow, and J. M. Baranowski, Appl. Phys. Lett. 79, 2576 (2001).

${ }^{6}$ S. Charbonneau, P. J. Poole, P. G. Piva, G. C. Aers, E. S. Koteles, M. Fallahi, J.-J. He, J. P. McCaffrey, M. Buchanan, M. Dion, R. D. Goldberg, and I. V. Mitchell, J. Appl. Phys. 78, 3697 (1995).

${ }^{7}$ H. H. Tan, J. S. Williams, C. Jagadish, P. T. Burke, and M. Gal, Appl. Phys. Lett. 68, 2401 (1996).

${ }^{8}$ H. H. Tan and C. Jagadish, Appl. Phys. Lett. 71, 2680 (1997).

${ }^{9}$ L. Fu, H. H. Tan, M. B. Johnston, M. Gal, and C. Jagadish, J. Appl. Phys. 85, 6786 (1999).

${ }^{10}$ P. J. Wellmann, W. V. Schoenfeld, J. M. Garcia, and P. M. Petroff, J. Electron. Mater. 27, 1030 (1998).

${ }^{11}$ T. Surkova, A. Patan, L. Eaves, P. C. Main, M. Henini, A. Polimeni, A. P. Knights, and C. Jeynes, J. Appl. Phys. 89, 6044 (2001).

${ }^{12}$ L. Fu, Ph.D. thesis, Australian National University, 2001.

${ }^{13}$ R. Leon, D. R. M. Williams, J. Krueger, E. R. Weber, and M. R. Melloch, Phys. Rev. B 56, 4336 (1997). 